\title{
SALUTO DEL PRESIDENTE DELLA VENERANDA FABBRICA DEL DUOMO
}

\author{
MARCO OROMBELLI
}

Desidero ringraziare l'Istituto Lombardo Accademia di Scienze e Lettere che ha voluto promuovere questo Incontro di cui abbiamo ben due nostri Cosiglieri che sono oratori, il prof Corradi Dell'Acqua e la prof.ssa Vismara.

Quando si parla del Duomo per noi è sempre una gioia, perché purtroppo oggi i milanesi se lo dimenticano e ben pochi sanno un po' le cose, quindi questa iniziativa ci rende anche un conforto morale perché vuol dire che le persone di cultura, le persone che sanno le cose, si dedicano, si ricordano del Duomo e fanno anche questo Incontro.

Quindi voglio rivolgere un saluto ai presenti, ringraziare il Presidente, il Vicepresidente che si è fatto animatore di questa iniziativa. Ringrazio con molta cordialità. 